Themenheft Nr. 40: CoViD-19 und die digitale Hochschulbildung. Irritationen, Einsichten und Programmatiken

Herausgegeben von Markus Deimann, Marios Karapanos und Klaus Rummler

\title{
Implikationen der Covid-19-Pandemie für digitale Lehre
}

\author{
Organisierte Freiheit als Veränderungsparadigma \\ Uwe Elsholz ${ }^{1}$ (D), Benedikt Fecher ${ }^{2}$ (D), Bronwen Deacon ${ }^{2}$ (D), Len Ole Schäfer ${ }^{1}$ (D) und \\ Melissa Laufer ${ }^{2}$ \\ ${ }^{1}$ FernUniversität in Hagen \\ ${ }^{2}$ Alexander von Humboldt Institut für Internet und Gesellschaft, Berlin
}

\begin{abstract}
Zusammenfassung
Der Artikel behandelt die Erfahrungen Lehrender mit den Bedingungen von Hochschullehre während der Covid-19-Pandemie. Wir berichten über erste Ergebnisse einer zweiphasigen Fokusgruppendiskussion, die wir zu Beginn und am Ende des Sommersemesters 2020 mit Lehrenden deutscher Hochschulen durchgeführt haben. Ziel der Studie ist es, organisationale Veränderungsprozesse in Bezug auf die Digitalisierung der Lehre zu analysieren. Die Covid-19-Pandemie wird dabei als ein Realexperiment verstanden, dass es ermöglicht, die seit vielen Jahren diskutierte digitale Hochschullehre explorativ zu untersuchen. Wir berichten dabei über Ergebnisse in drei unterschiedlichen Dimensionen. Diese betreffen Aspekte der Veränderungsbereitschaft Lehrender, Fragen der Führung sowie der Bereitstellung von Ressourcen seitens der Hochschulen. Aus den Befunden leiten wir Schlussfolgerungen für die (zukünftige) Organisation von Hochschullehre ab. Zentral ist dabei das Prinzip der "Organisierten Freiheit». Dieses Prinzip verweist auf typische Veränderungslogiken auf unterschiedlichen Ebenen der Hochschule. Mit dem Beitrag sollen damit Hinweise für die Hochschulentwicklung gewonnen werden in Bezug auf die nachhaltige Gestaltung digitaler Lehr- und Lehrinnovationen.
\end{abstract}

Implications of the Covid-19 Pandemic for Digital Teaching. Organised Freedom as a Paradigm of Change

\footnotetext{
Abstract

The article investigates university instructors' teaching experiences during the Corona pandemic. We report on the initial results of a two-phased study that was conducted with instructors working at German higher education institutions. Specifically, data were
} 
collected at the beginning and the end of the summer semester 2020 through focus group discussions. This study analyses the organisational change processes that occurred across universities in relation to the digitalisation of teaching. The Corona pandemic provoked a real-life experiment, making it possible for instructors to explore digital teaching, a topic that has been greatly discussed in recent years. In the findings, we discuss three different dimensions that emerged: instructors' willingness to change, questions of leadership and the allocation of institutional resources. Drawing on these findings, we derive conclusions for the (future) organisation of university teaching and discuss the principle of "organised freedom». This principle highlights typical change logics within different levels of the university. These findings contribute to our understanding of higher education development and the sustainable design of digital teaching and learning innovations.

\section{Die Lehre während Corona als unfreiwilliges Realexperiment}

Wie organisiert sich Lehre, wenn es die Universität - als Ort - nicht gibt? Diese Frage scheint rein hypothetischer Natur, beschreibt aber treffend die Situation der Hochschullehrenden als die Corona-Pandemie im Frühjahr 2020 Deutschland erreichte und klar wurde, dass Universitäten ihren Präsenzbetrieb einstellen müssen. Im Gegensatz zur Situation an Schulen schien die unfreiwillige Umstellung auf digitale Lehre relativ gelungen, immerhin wurde ein Grossteil der Lehrveranstaltungen trotz der Schliessungen der Hochschulen durchgeführt. Dabei gab es unter den Lehrenden durchaus auch skeptische Stimmen: Ein offener Brief zur «Verteidigung der Präsenzlehre» wurde von mehr als 6000 Hochschullehrenden unterschrieben (www.präsenzlehre.com). Eine andere Initiative, mit beinahe 16.000 Unterschriften, forderte ein «Nichtsemester» und begründen dies damit, dass das Semester «mehr [verlangt] als E-Learning Kompetenzen» (Villa Braslavsky et al. 2020). Es lässt sich feststellen, dass die Lehre organisiert wurde und stattfand, obwohl die Universität als Ort in der akuten Pandemiezeit nicht zur Verfügung stand. Es zeigt sich allerdings auch, dass die abrupte Umstellung auf digitale Lehre bei Lehrenden Irritationen verursachte und diese insbesondere die Lehrorganisation betreffen.

Vor diesem Hintergrund behandelt der Beitrag die Organisation von Lehre während der Corona-Pandemie. Lehrorganisation wird hier als Teil jener Organisation verstanden, die der Hochschule - neben Forschung und Transfer - als Funktion zugeschrieben wird und welche die zur Bewerkstelligung von Lehre notwendigen Koordinierungsprozesse, personellen Kapazitäten und Ressourcen an Hochschulen berührt. Die Notwendigkeit der konstruktiven Reflexion über organisationale Fragen digitaler Lehre wurde von prominenten Stellen mehrfach formuliert, etwa von einer Arbeitsgruppe im Hochschulforum Digitalisierung (Wannemacher et al. 2016) oder zuletzt vom Wissenschaftsrat in einem Positionspapier (Wissenschaftsrat 2021). Obgleich es vielversprechende Ansätze gibt, die Lehr- und Organisationsentwicklung an 
Hochschulen miteinander in Beziehung zu setzen (z. B. Brahm et al. 2016; Bosse et al. 2020), sind - mit wenigen Ausnahmen (z. B. Kosmützky 2018; Gilch et al. 2019; Getto et al. 2018; Graf-Schlattmann et al. 2019) - Organisationsbedingungen für digitale Lehre an deutschen Hochschulen wenig erforscht. Hier sehen wir eine Forschungslücke und streben eine Erweiterung des wissenschaftlichen Diskurses über digitale Lehre um eine organisationale Dimension an. Hierzu liegen zwar erste quantitative Untersuchungen vor (vgl. Winde et al. 2020), doch erweitern wir diese um eine qualitative Perspektive.

Gestützt wird die nachfolgende Argumentation durch die Auswertung von qualitativen Befragungen von insgesamt 27 Lehrenden deutscher Hochschulen, die wir in zwei Phasen zu Beginn und am Ende des Sommersemesters 2020 durchgeführt haben. Die zentrale Schlussfolgerung bezogen auf die «Organisierte Freiheit» bezieht sich auf ein übergreifendes Konzept in den Diskussionen und verweist auf typische Veränderungslogiken auf unterschiedlichen Ebenen der Hochschule (Lehrende, Führung, Infrastruktur). Lehrende wünschen sich demnach sichere Rahmenbedingungen für die freie Gestaltung der Lehre. Dieser Anforderung aus Lehrendenperspektive gehen wir im Verlauf des Beitrags weiter nach und explizieren, was dieses Prinzip bedeutet.

\section{Die Studie «Bewältigung der Krise: Organisation der Lehre in unsicheren Zeiten»}

Die hier zu Grunde liegende Studie behandelt organisationale Veränderungsprozesse an deutschen Hochschulen durch die pandemiebedingte Digitalisierung. Sie ist im Rahmen des Forschungsprojekts «Organisationale Adaptivität im deutschen Hochschulkontext» im Forschungsschwerpunkt $D^{2} L^{2}$ der FernUniversität in Hagen entstanden. Um die Umstellung der Lehre bereits zu einer frühen Phase des Sommersemesters 2020 zu erfassen, wurde die Studie sehr kurzfristig durchgeführt und dient der Exploration organisationaler Bedingungen für die Implementierung von Lehr- und Lerninnovationen aus der Perspektive der Lehrenden. Hierzu wurden zu zwei Zeitpunkten Daten erhoben: Im Rahmen von (1) 13 Fokusgruppen von April Mai 2020 und (2) einer darauf aufbauenden Online-Umfrage von Oktober - November 2020. Zunächst führten wir 13 ca. 60-minütige Online-Fokusgruppen-Diskussionen mit Lehrenden an Hochschulen $(n=27)$ durch. Bei der Auswahl der Diskutierenden wurde darauf geachtet, dass sie unterschiedliche Disziplinen, Hochschultypen und Bundesländer vertreten, um einem zu engen Fokus vorzubeugen. Die kleine Anzahl an Teilnehmenden pro Fokusgruppe wurde bewusst gewählt, um eine vertiefte Diskussion auch online zu ermöglichen (Morgan 1996, 129ff.). Die Fokusgruppendiskussionen wurden anhand eines semi-strukturierten Leitfadens durchgeführt, der im Laufe der Erhebung - und mit neu aufkommenden Erkenntnissen und Hypothesen 
- angepasst wurde. Die Teilnehmenden wurden nach ihrem Umgang und Erfahrungen mit digitalen Tools, der Kollaboration und Koordination unter Kolleginnen und Kollegen sowie nach der Unterstützung durch die Hochschulleitung und anderen zentrale Einheiten befragt. Die Gespräche wurden nach Einwilligung der Befragten aufgezeichnet und vollständig transkribiert. Mithilfe der Software MAXQDA wurden die Daten im Zusammenwirken von deduktiven und induktiven Codes inhaltsanalytisch ausgewertet (Reichertz 2014, 123ff.). Im Rahmen einer kommunikativen Validierung wurden die Befragten im Juli 2020 zu einem Online-Meeting eingeladen, bei dem vorläufige Ergebnisse der ersten Untersuchungsphase vorgestellt und diskutiert wurden.

Am Ende des Sommersemesters wurden die Teilnehmenden der Fokusgruppen erneut kontaktiert und mit einem Fragebogen um ihre Einschätzung gebeten. Der Fragebogen enthielt sowohl geschlossene als auch offene Fragen. Das Ziel dieses zweiten Schrittes war es, festzustellen, inwiefern sich die Umstände - speziell in Bezug auf die organisationale Unterstützung - seit der Umstellung auf rein digitale Lehre verändert haben. Wichtig war uns hier, auch Veränderungen aufzunehmen, die sich im Verlauf des Semesters ergeben haben.

Aufgrund ihres explorativen Charakters der sich fortwährend verändernden Umstände für die Lehre, besitzen die vorgestellten Ergebnisse zwangsläufig explorativen Charakter. Sie können jedoch dabei behilflich sein, die Lehrorganisation während der Covid-19-Pandemie besser zu verstehen und letztlich Schlüsse für die Organisation digitaler Lehre auch nach der Pandemie zu ziehen.

\section{Untersuchungsergebnisse}

Die Ergebnisdarstellung erfolgt entlang von drei prominenten Themen, die in den Fokusgruppengesprächen als relevant deutlich wurden und die unterschiedliche Ebenen berühren: (1) die Veränderungsbereitschaft der Lehrenden, (2) Fragen der formellen und informellen Führung und (3) Aspekte der materiellen und immateriellen Ressourcen. In den Fokusgruppen wurde in Bezug auf diese Themenfelder ein breites Spektrum an (teilweise durchaus gegensätzlichen) Erfahrungen und Reaktionen deutlich, die in den Abschnittsüberschriften festgehalten sind. Wir stellen im Folgenden zentrale Beobachtungen entlang der drei Themen vor und setzen diese jeweils in Beziehung zu relevanten organisationssoziologischen Konzepten.

\subsection{Innovationsfreude versus Veränderungsskepsis}

Eine erste Dimension unserer Befragung betrifft das Thema der Veränderungsbereitschaft Lehrender, die sich sehr kurzfristig mit der Umstellung auf digitale Lehrformate konfrontiert sahen. In den Gesprächen berichteten die Lehrenden offen über ihren 
Umgang mit der Umstellung des Lehrbetriebs und es wurden durchaus widersprüchliche Positionen der Lehrenden erkennbar, die sich zwischen grosser Offenheit und zurückhaltender Skepsis bewegen. Eine Art Innovationsfreude äusserte sich in Aussagen der Lehrenden, die die Krisensituation als «große Chance» beschrieben, unter anderem für die Qualitätsentwicklung der digitalen Lehre, die Mobilität von Lehrenden und Studierenden und die (solidarische) Gemeinschaft der Lehrenden. Lehrende berichteten unter anderem über die Freude am Ausprobieren neuer Werkzeuge und Formate. Sie beschrieben die Ausnahmesituation als ein willkommenes Experimentierfeld und eine Möglichkeit, sich neue Kenntnisse anzueignen und ihre Lehre zu aktualisieren. Folgendes Zitat eines Befragten, der an einer Kunsthochschule lehrt, illustriert dies:

«[...] [Ich] habe das Gefühl, dass das überall als große Chance wahrgenommen wird und spielerisch damit umgegangen wird. Dass alle Kolleginnen und Kollegen das als Herausforderung annehmen. Und das hat erstmal, würde ich sagen, würde ich erwarten, positive Folgen für die Qualität der Lehre, weil die Leute Veranstaltungen, die sie seit vielen Jahren machen, auf einmal überdenken, aktualisieren, übersetzen in andere Formate.» (FG_1, Pos. 7)

Eine positive Einstellung zur Veränderungssituation zeigt sich auch an der Wahrnehmung der Krise als eine Stärkung des Gemeinschaftsgefühls unter den Lehrenden:

«Das ist ein ganz besonderer Spirit, der bei den Kolleg_innen entstanden ist. Wirklich diesem Gemeinschaftsgefühl ähnlich, wie [Name] das auch erzählt hat. So eine Aufbruchstimmung, also wir haben jetzt eine Riesen-Herausforderung vor der Brust, aber wir rocken das gemeinsam.» (FG_1, Pos. 40)

Eine ähnlich schon fast euphorische Haltung zur pandemiebedingten Umstellung der Lehre äusserte sich insbesondere zu Beginn der Krise bei einem überwiegenden Teil der Befragten. Ein Lehrender an einer Fakultät für Medizindidaktik und Lehrentwicklung beschrieb dies als «Honeymoonphase» (der jedoch später auch eine depressive Phase folgte).

Auch wenn die Selbstselektion der von uns rekrutierten Teilnehmenden der Studie vermutlich zu einem Übergewicht stärker medienaffiner Befragter geführt hat, wurde in den Gesprächen jedoch auch eine durchaus anzutreffende Skepsis deutlich. Ein Musiker einer Kunsthochschule beschrieb dies sehr bildlich:

«Ja, es gibt erstmal die Angst und die Unsicherheit, die man bekommt, wenn man mit sehr großer Sicherheit ein Auto gefahren ist und plötzlich erfährt man, nee, dieses Auto fährt jetzt ganz anders. Wir wissen nicht genau, wie man damit umgeht. Man muss das auch kennenlernen, wir müssen die Formate entwickeln und was der Kollege [Name] sagte, es nimmt alles viel, viel mehr Zeit.» (FG_7, Pos. 24) 
Viele Befragten äusserten Bedenken über die Einbeziehung von Lehrenden, die nicht über die notwendigen Kompetenzen für die digitale Lehre verfügen und abgehängt werden könnten. Ein Lehrender aus dem Fachbereich Wirtschaft einer Fachhochschule beschrieb die anfängliche Reaktion, als feststand, dass die Lehre online durchgeführt wird, als Zustand «größte[r] Nervosität und allerhöchste[r] Not». Eine weitere Befragte aus den Theaterwissenschaften macht besonders auf die Situation von Lehrenden und Studierenden mit Betreuungsaufgaben aufmerksam, die durch die zeitweise Schul- und Kitaschliessungen doppelt belastet wurden:

«Also die größte Herausforderung ist die Kinderbetreuung. Es ist sozusagen eine Sache mit dem wir an dieser Uni und an vielen anderen relativ allein sind. Es gibt ein bisschen einen Riss durch die Situation. Diejenigen Personen, die keine Kinder im Kindergartenalter haben sind relativ gut bestellt. Bei anderen, gerade bei vielen Kolleginnen, die in der Qualifikationsphase sind, enden Karrieren gerade so bisschen.» (FG_9, Pos. 20)

Neben Sorgen vor technischer Überforderung oder hinsichtlich der eigenen Arbeitsbedingungen im Home Office berichteten viele Lehrende zudem über Momente der Ernüchterung, etwa in Bezug auf den gestiegenen Arbeitsaufwand, der fast durchgängig als grosse Herausforderung beschrieben wurde. Darüber wurden auch Unklarheiten bzgl. der Anerkennung auf das Lehrdeputat oder die Abnahme von Prüfungen als problematisch benannt.

Insbesondere jene Personengruppen empfinden die Situation als positiv, die bereits über Erfahrungen in der digitalen Lehre verfügen und nun weniger Hindernisse als üblich in der Umsetzung ihrer Vorstellungen verspürten. Dieser Befund deckt sich auch mit vorangegangenen Studien (Long, Cummins, und Waugh 2019; Lai, Hsiao, und Hsieh 2018; King und Boyatt 2014). Die skeptischen Einschätzungen der Situation wurden zumeist auf soziale Herausforderungen und unklare administrative Prozesse bezogen. Auch das deckt sich mit Erkenntnissen weiterer Studien (Lin und Cantoni 2018; Awidi und Cooper 2015; Schneckenberg 2010) und legt nahe, dass grundlegende Sicherheiten von vielen Lehrenden als Bedingungen für Veränderungen in der Lehrorganisation betrachtet werden. Allerdings stehen unsere Ergebnisse teilweise im Widerspruch zu Studien, die von Veränderungsresistenzen von Hochschulmitgliedern (Agasisti et al., 2018; Kalfa et al. 2018; Ryan 2012) berichten. Vielmehr überwogen in unserer Befragung deutlich die Momente der Freude und der Innovation - was sich auch in der (vor allem im Vergleich zum Schulsektor) relativ reibungslosen Umstellung auf digitale Lehre im Sommersemester 2020 zeigte. 


\subsection{Formelle und informelle Führung}

Eine zweite sehr instruktive Dimension unserer Befragung betrifft das Thema Führung. Obwohl wir im Gesprächsleitfaden Führung insbesondere in Bezug auf die Hochschulleitung aufgenommen haben, wurde seitens der Befragten auf zwei unterschiedliche Konzepte von Führung Bezug genommen. Zum einen die formelle Führung, die an die offiziellen Strukturen und Positionen der Organisation geknüpft ist (z. B. Präsident, Rektor, Dekan, IT-Leitung), und zum anderen die informelle Führung, die auch jenseits formaler Strukturen und Positionen entstehen kann.

Die formelle Führung bezieht sich aus Sicht der Lehrenden auf das Schaffen der Bedingungen für die Bewerkstelligung der Lehre und wird insbesondere als eine Aufgabe der Hochschulleitung wahrgenommen. Diese soll grundlegende operative und logistische Standards setzen und Unsicherheiten ausräumen, um damit die Lehrgestaltung zu ermöglichen. Als Beispiele dieser Standards, wurden in den Gesprächen die Abnahme von Prüfungen online, der Datenschutz und Lizenzlösungen bei der Verwendung von Software oder die Anerkennung des Mehraufwands für das Lehrdeputat genannt. In vielen Fällen liess sich beobachten, dass sich Lehrende in der Durchführung der Lehre behindert sahen, wenn diese Fragen ungeklärt blieben, wie folgendes Zitat einer Lehrenden einer Fachhochschule aus dem Bereich Wirtschaft vermittelt:

«Aber es existieren noch so viele Fragezeichen. Ich habe es gerade schon gesagt, bezüglich der Tools, der Anwendungen, die wir benutzen sollen, gibt es noch keine einheitliche Regelung. Ähnlich geht es uns jetzt in Bezug auf die Planung der Prüfungen und Klausuren. Da gibt es jetzt, also zumindest bis zum Stand heute, auch noch keine klare Aussage, wie normale klassische Klausuren jetzt in Form einer E-Klausur stattfinden können. Welche Anwendungen da geeignet sind, welche Vorsichts- und Sicherheitsmaßnahmen getroffen werden müssten, Datenschutzmaßnahmen getroffen werden mussten.»(FG_11, Pos. 15)

Zudem wurden der Hochschulleitung soziale Verantwortung zugeschrieben, die sich neben einer Überforderung von Lehrkräften auch auf die Arbeitsbedingungen prekär Beschäftigter beziehen. Einige der Befragten wünschten sich seitens der formalen Leitung zusätzliche personelle Ressourcen, Fortbildungen und kontinuierlichen IT-Support, der teilweise auch gewährleistet wurde (z. B. in Form von «escouts», technisch versierte Studierende, die den Lehrenden zur Seite standen).

Viele der Befragten äusserten ihre Unzufriedenheit mit der Führung durch die Hochschulleitung, insbesondere zu Beginn der Pandemie; sie brachten ihr in einigen Fällen - durch die Schwierigkeiten des schnellen Umstiegs auf digitale Lehre - auch Verständnis entgegen. In der zweiten Phase der Studie berichten einige der 
Lehrenden sogar, dass sich die formelle Führung im Laufe des Semesters verbessert habe, in dem Sinne, dass offene Fragen seitens der Leitung geklärt oder zumindest angegangen wurden.

Die informelle Führung ist dagegen entkoppelt von formalen Strukturen und tritt situativ auf. Aus Sicht der Lehrenden bezieht sie sich auf die Gestaltung der Lehre und tritt in Momenten, in denen die Initiative einzelner Lehrender Spill-Over-Effekte erzeugen. In unseren Gesprächen liessen sich einige Fälle dafür finden, etwa Lehrende, deren Methoden für die Online-Lehre auch von Kolleginnen und Kollegen angewandt werden, Unterstützungsnetzwerke initiiert haben oder beratend für andere Lehrende wirkten. In einem Zitat eines Lehrenden einer Fachhochschule im Bereich Wirtschaft wird dies besonders deutlich:

«Wir öffnen jetzt unsere Tore und sagen eben zu Kollegen, die sich schwerer tun, vielleicht auch, weil sie eben die Kompetenz nicht haben, dass wir sie gerne einladen zu uns. Und wir haben ja schon, wie soll ich sagen, so ein First Aid Kit zusammengestellt. Das ist eine Spiegelreflexkamera mit einem Richtmikrofon. Sie müssen es nur anmachen. Um den Rest kümmern wir uns. Also, wir versuchen das wirklich auch in die anderen Fachbereiche ausstrahlen zu lassen, was wir uns aufgebaut haben.» (FG_13, Pos. 50)

Oftmals begründet sich informelle Führung damit, dass Lehrende bereits über Kompetenzen für die digitale Lehre verfügten und diese nun besonders gefragt waren. Ein Lehrender, der bereits am Aufbau eines digitalen Moduls in der medizinischen Ausbildung mitgearbeitet hat, berichtete, dass seine Methoden nun von weiten Teilen der Fakultät genutzt werden. Ein anderer, der gerade seine Promotion im Feld der Online-Didaktik abgeschlossen hatte, berichtete, dass er nun regelmässig von Kolleginnen und Kollegen konsultiert würde und seine Expertise sowohl von diesen und auch von der Hochschulleitung deutlich mehr geschätzt würde. Auffallend ist, dass vereinzelt auch Studierenden Führungskompetenzen zugeschrieben werden dahingehend, dass Lehrende durch Studierende Beratung erfahren oder diese Lehrmaterialien co-kreieren.

In Bezug auf die beiden Formen der Führung, die wir in unseren Gesprächen beobachten konnten, fällt auf, dass sie sich auch funktional unterscheiden, dahingehend, dass der formellen Führung Aufgaben zugeschrieben werden, die sich auf die äusseren Lehrbedingungen beziehen. Diese Beobachtung deckt sich mit Beschreibungen der administrativen Führung (vgl. Uhl-Bien et al. 2007; Yukl 2005; Ng'ambi et al. 2013). Demnach weist die administrative Führung Ressourcen zu, managt Krisen und Konflikte und steuert die Organisationsstrategie (siehe auch Mumford, BedellAvers, und Hunter im Druck). Der informellen Führung werden Aufgaben zugeschrieben, die sich auf die Gestaltung der Lehre beziehen. Dies entspricht dem Konzept der adaptiven Führung, welches sich auf kreative und lernende Handlungen bezieht 
(Uhl-Bien et al. 2007; Billot et. al. 2013; Ng'ambi et al. 2013; Tsai et al. 2019). Auch wenn mehrheitlich auf die Gestaltung der Lehre bezogen, lassen sich in den Diskussionen Beispiele für adaptive Führung auf den formalen Leitungspositionen finden. Interessant und relevant für die weitere Diskussion und Organisation hochschulischer Lehre ist nur gerade das Zusammenspiel adaptiver Führung und administrativer Führung.

\subsection{Materielle und immaterielle Ressourcen}

Die dritte hier berichtete Dimension bezieht sich auf zwei unterschiedliche Formen von Ressourcen, die für die hochschulische Lehre vonnöten sind. Diese lassen sich in materielle und immaterielle Ressourcen unterscheiden, die zur Bewerkstelligung von Lehre genutzt werden. Materielle Ressourcen beziehen sich auf physische und ökonomische Infrastrukturen der Hochschule, wohingegen immaterielle Ressourcen insbesondere kognitive und digitale Infrastrukturen umfassen. Materielle Ressourcen beziehen sich auf physische Artefakte, die aufgrund der Einstellung des Präsenzbetriebs nicht oder nur sehr bedingt zur Verfügung stehen. In Fällen besonderer Abhängigkeit von materiellen Ressourcen hatte dies zur Folge, dass Lehre nicht oder nur teilweise organisiert werden konnte. In den Gesprächen berichteten viele Lehrende über materielle Grenzen der Digitalisierung der Lehre, wobei dies stark von der wissenschaftlichen Disziplin abhängig war. Die Einschränkungen bezogen sich unter anderem auf Räumlichkeiten (z. B. ein Proberaum für den Orchesterunterricht), Instrumente (z. B. Labore für die medizinische Lehre) und Maschinen ( $z$. B. Werkbänke in der Arbeitslehre). Da Lehre in einigen Fachbereichen stark von materiellen Ressourcen abhängig ist, musste die Lehre in diesen Fällen oftmals entfallen, wie folgendes Zitat verdeutlicht:

«Arbeitslehre/Technik ist ein Studiengang, der sehr viel mit Fachpraxis arbeitet. Das heißt, bei uns sollen die Studierenden später ja Hauswirtschaftslehre, unter anderem, unterrichten. Und auch in Werkstätten arbeiten mit Schülerinnen und Schülern. Das bedeutet, dass sie natürlich bei uns auch in Werkstätten arbeiten müssen. Also, sie müssen an den Geräten arbeiten. Und später überhaupt Schülerinnen und Schüler einweisen zu können in die Geräte. Und das sind natürlich Veranstaltungen, die wir jetzt so, in dem Sinne, nicht anbieten können.» (FG_12, Pos. 15)

In der Medizin konnten materielle Grenzen überwunden werden, in dem Software entwickelt wurde, die einen virtuellen Patienten simulierte. Doch auch die Software konnte die fehlende Präsenz nicht vollumfänglich ersetzen. Des Weiteren wies ein Interviewpartner darauf hin, dass das Lernen anhand einer Software mit einer Standardisierung des Wissens einherging, die sich im Falle von Präsenzveranstaltungen und der Wissensvermittlung durch Ärzte nicht ereignet hätte. 
«Weil in dem Bereich Patientensicherheit, Mitarbeitersicherheit geht es sehr viel auch um Simulationen. Und da gibt es auch relativ viel im Bereich Virtual Patient [...] Haben wir versucht, die Lehrinhalte so gut wie möglich abzubilden. Was ein bisschen ein Problem ist, weil diese Lernplattform vor allem daran ausgerichtet ist, Personen nach so einer Art Karteikartensystem, Wissen zu vermitteln. Das ist auch quer verlinkt, das ist eine sehr innovative Plattform. Aber die bildet eigentlich nicht den Wissensstand für einen Arzt ab oder für einen zukünftigen Arzt. [...] Sondern sie bildet eher das Wissen ab, was man für diese große Prüfung braucht.» (FG_11, Pos. 9)

Die immateriellen Ressourcen beziehen sich auf zumeist digitale Infrastrukturen, die von den Lehrenden verstärkt genutzt werden mussten und die neue, dezentrale Formen der Organisation bedingten. Wir beobachteten bei verschiedenen Befragten beispielsweise verstärkt die Initiierung dezentraler (Selbsthilfe-) Netzwerke mittels Videoplattformen und den Aufbau von dezentralen Repositorien für Arbeitsmaterialien. Dies verdeutlicht die folgende Reaktion eines Lehrenden im Fach Data Science an einer privaten Hochschule:

«Wunderbar ja, also wirklich perfekt. Der Austausch ist, bei uns passiert glaube ich ganz viel Austausch direkt auch auf der Kollegenebene. Also sicherlich auch, dass der Direktor das auch mal irgendwie mal macht. Aber eigentlich passiert schon, die meisten Innovationssachen passieren direkt unter den Kollegen, dass jeder was austauscht und man sich irgendwie regelmäßig in VideoCalls sich mal kurz austauscht: Was funktioniert gerade, was funktioniert gerade nicht? Und wir haben einfach ein gutes Team an Leuten, die Videoschnitt können, die irgendwie sich um die Website, Social Media, alle diese Sachen kümmern. Also das ist wunderbar.» (FG_2, Pos. 24)

In der qualitativen Untersuchung wurden materielle und immaterielle Ressourcen beobachtet, die zur Gestaltung der Lehrorganisation notwendig sind. Während materielle Ressourcen meist mit der zentralen Ebene der Hochschule bzw. letztlich der Hochschulleitung verknüpft sind, deuten immaterielle Ressourcen auf die für die Lehrorganisation charakteristische dezentrale Ebene hin. Diese Perspektive der Dezentralität ist fest in der Organisationsforschung verankert (vgl. Frost und Hattke 2013) und begreift die einzelnen Elemente lose miteinander gekoppelt, nicht zentral koordiniert und nicht kontrolliert (vgl. Weick 1976; Tippelt 2009). Das Zusammenwirken von zentralen und dezentralen Ressourcen erscheint damit letztlich entscheidend für eine erfolgreiche Gestaltung von Innovationen und digitaler Lehre. 


\section{Organisierte Freiheit als Organisationsprinzip}

Wir haben zuvor Befunde in drei unterschiedlichen Dimensionen dargestellt, die wir abschliessend zueinander in Bezug setzen. Dabei stehen vor allem Schlussfolgerungen für die Organisation Hochschule im Zentrum. Aus dem Gegensatz von Innovationsfreude versus Veränderungsskepsis lässt sich schlussfolgern, dass als Aufgabe der Hochschule gesehen werden kann, Lehrenden «Freiheit zur...» und zugleich «Sicherheit durch...» jeweils geeignete Massnahmen zu gewähren. Aus den Ergebnissen zur formeller und zur adaptiven Führung ergeben sich ähnliche Aspekte, indem formelle Führung gewisse (Handlungs-)Sicherheiten der Lehrenden gewährleisten muss, zugleich aber Raum für adaptive Führung bleiben sollte. Hinsichtlich der Frage der Ressourcen ist ebenfalls zu unterscheiden zwischen offiziell zur Verfügung gestellten und damit häufig materiellen Ressourcen, die Lehrenden Sicherheit geben und auf die sie zurückgreifen können. Informelle Netzwerke, häufig unter Kolleginnen und Kollegen, ermöglichen jedoch darüber hinaus stärker Momente der Freiheit und der Innovation.

Die verschiedenen Befunde verweisen damit in der Zusammenschau auf ein gemeinsames Grundprinzip zwischen Sicherheit und Freiheit, dass wir als «organisierte Freiheit» bezeichnen. Damit knüpfen wir auch an Ergebnisse von Graf-Schlattmann et al. (2020) an, die die Themen Freiräume und Professionalität als wesentliches Moment erfolgreicher Digitalisierung von Hochschullehre herausgearbeitet haben.

Im Hinblick auf die Frage, inwiefern «organisierte Freiheit» ein essentielles Organisationsprinzip hochschulischer Lehre sein kann oder sollte, erscheinen die Besonderheiten von Hochschulen als Organisation (Wilkesmann und Schmid 2012) von grosser Relevanz. Diese Besonderheiten etwa im Vergleich zu Schulen oder Unternehmen begründen nicht zuletzt auch das von uns als Schlussfolgerung vorgestellte Organisationsprinzip. Hochschulen sind als Organisationen damit beauftragt, Wissen zu produzieren, zu zertifizieren und zu vermitteln. Sie sind, wie Eaton und Stevens (2019) es nennen, eine sonderbare («peculiar») Organisation. Die akademische Freiheit befindet sich im Herzen der institutionellen Gestaltung der Universität (vgl. Altbach 2001, 205) und gerade die Idee der Lehrfreiheit ist dabei historisch verwurzelt und essentiell in Deutschland (vgl. Enders 2001). Sie besteht in der «[...] Freiheit der Wahl der Themen und die Freiheit der Wahl der Behandlung der Themen.» (Stichweh 2016, 27). Die Lehre wird in dieser idealtypischen Vorstellung vom Forschergeist informiert und durchdrungen. Der/die einzelne Lehrende sieht sich jedoch auch den Erwartungen der Administration der Universität ausgesetzt. Diese wirkt als Rahmung, die der professionellen Autonomie des Lehrenden gegenübersteht und zeichnet sich durch eine Ressourcen- und Kontrollfunktion aus, die die wissenschaftliche Praxis umgibt. Wissenschaftlerinnen und Wissenschaftler sind i. d. R. skeptisch gegenüber der formalen und bürokratischen Ordnung der Institution der Universität und treten für seine Autonomie ein (vgl. Stichweh 2016, 31). 
Der klassische Gegensatz zwischen Zentralität und Dezentralität, zwischen formaler und informeller Struktur, zwischen Verwaltung und professioneller Autonomie spiegelt sich auch in der Digitalisierungsdebatte. Freiheiten und Gestaltungsspielräume von Lehrenden, die zu Innovations- und Experimentierfreude in der Lehre führen können - wie wir es besonders zu Beginn der Pandemie beobachten konnten - können durch Strukturen auf Organisationsseite unterstützt und gefördert werden. Aus der Perspektive der Organisation kommt es insbesondere darauf an, mit unterstützenden Massnahmen die Innovationsbereitschaft aufrechtzuerhalten, welche in der ersten Phase der Umstellung durch geringe externe Einwirkung auf den Freiheitsspielraum der Lehrende entstanden ist. Gleichermassen kann so Skepsis begegnet und Ängste reduziert werden sowie Einfluss auf Einstellung und Kompetenz genommen werden. Da Angst vor Veränderung die Wahrnehmung von Freiheit einschränkt, stellt die Unterstützung der Organisation als ein Sicherheitsnetzwerk eine Bedingung für Freiheit dar.

Nicht die Freiheit als Solches ist dabei das Ziel, sondern die Freiheit muss organisiert sein und sie kann und sollte organisational unterstützt werden. Dazu gehört einerseits die ausreichende Bereitstellung technischer Infrastruktur, die in manchen Fächern und Disziplinen (wie etwa in der Medizin, Kunst oder Musik) durchaus aufwändiger ist. Dazu gehört aber andererseits auch die gezielte Förderung entsprechender Kompetenzen der Lehrenden. Und zu diesen Kompetenzen gehört nicht nur die Handhabung entsprechender Soft- und ggf. Hardware, sondern dazu gehört auch die Entwicklung pädagogischer Professionalität (vgl. Schütz et al. i. E.). Erst durch eine entsprechende Kompetenzentwicklung werden Lehrende in die Lage versetzt, die Freiheit auch zu nutzen für die eigenständige Gestaltung digitaler Lehre. Organisierte Freiheit heisst demnach Kompetenzen zu erkennen, zu fördern und Rahmenbedingungen zu schaffen, damit diese auch fruchtbar eingesetzt werden im Sinne der insbesondere von Wilkesmann (2012) fokussierten Self-determination theory. Lehren an Hochschulen ist nicht nur eine individuelle Frage, sondern auch organisational gerahmt. Der nachhaltige Einsatz von Medien in der Lehre ist daher ebenso an organisationale Bedingungen gekoppelt. Auch wenn der Terminus «Lernende Organisation» schon etwas aus der Mode gekommen ist, könnte eine «organisierte Freiheit» hinsichtlich der Lehrorganisation genau dazu einen wesentlichen Beitrag leisten.

Wir sind uns durchaus bewusst, dass in die Gestaltung von digitaler Hochschullehre auch andere Stakeholder einbezogen sind - Studierende, die Hochschulleitung und letztlich auch die Gesellschaft als Ganzes. Insbesondere die Studierendenperspektive ist für eine reflektierte medien- und hochschuldidaktische Perspektive unerlässlich. Sie stand jedoch nicht im Fokus unserer Studie und ist daher durch andere Untersuchungen in eine Gesamtschau einzubeziehen. Mit der Fokussierung der Perspektive der Lehrenden und dem Prinzip der «organisierten Freiheit» wurde bewusst 
eine Perspektive eingenommen und genauer ausgeschildert, die wir damit im Diskurs um die zukünftige Gestaltung von Hochschullehre stark machen wollen, damit nicht ausschliesslich rechtliche, technische oder administrative Normierungen zu Bestimmungsfaktoren werden.

\section{Literatur:}

Agasisti, Tommaso, Giuseppe Catalano, und Angelo Erbacci. 2018. «How Resistance to Change Affects the Implementation of Accrual Accounting in Italian Public Universities: A Comparative Case Study». International Journal of Public Administration 41 (12): 946-56. https:// doi.org/10.1080/01900692.2017.1301469.

Altbach, Philip G. 2001. «Academic Freedom: International Realities and Challenges». Higher Education 41 (1): 205-19. https://doi.org/10.1023/A:1026791518365.

Awidi, Isaiah T., und Martin Cooper. 2015. «Using Management Procedure Gaps to Enhance ELearning Implementation in Africa». Computers \& Education 90 (Dezember): 64-79. https:// doi.org/10.1016/j.compedu.2015.08.003.

Bosse, Elke, Grit Würmseer, und Uwe Krüger. 2020. «Lehrentwicklung als organisationaler Veränderungsprozess». Zeitschrift für Hochschulentwicklung 15 (4): 135-56. https://doi. org/10.3217/zfhe-15-04/08.

Brahm, Taiga, Tobias Jenert, und Dieter Euler. 2016. «Pädagogische Hochschulentwicklung als Motor für die Qualitätsentwicklung von Studium und Lehre». In Pädagogische Hochschulentwicklung, 19-36. Springer. https://doi.org/10.1007/978-3-658-12067-2_2.

Enders, Jürgen. 2001. «A chair system in transition: Appointments, promotions, and gatekeeping in German higher education». Higher education, 41(1), 3-25. https://doi. org/10.1023/A:1026790026117.

Frost, Jetta, und Fabian Hattke. 2013. «University Commons. Kollektivressourcen als alternative Steuerungsperspektive für das Hochschulmanagement». Hochschulmanagement. 8 (2/3): $35-40$

Getto, Barbara, Patrick Hintze und Michael Kerres. 2018. «(Wie) Kann Digitalisierung zur Hochschulentwicklung beitragen?» In Digitalisierung und Hochschulentwicklung. Proceedings zur 26. Tagung der Gesellschaft für Medien in der Wissenschaft e.V. Münster, herausgegeben von Barbara Getto, Patrick Hintze und Michael Kerres. Münster, New York: Waxmann. https://doi.org/10.25656/01:16983.

Gilch, Harald, Anna Sophie Beise, René Krempkow, Marko Müller, Friedrich Stratmann, und Klaus Wannemacher. 2019. «Digitalisierung der Hochschulen: Ergebnisse einer Schwerpunktstudie für die Expertenkommission Forschung und Innovation». Research Report 14-2019. Studien zum deutschen Innovationssystem. https://www.econstor.eu/handle/10419/194284. 
Graf-Schlattmann, Marcel, Dorothee M. Meister, Gudrun Oevel, und Melanie Wilde. 2019. «Digitalisierungsstrategien auf dem Prüfstand - eine empirische Untersuchung auf Basis der Grounded-Theory-Methodologie an deutschen Hochschulen». In Teilhabe in der digitalen Bildungswelt, herausgegeben von Jörg Hafer, Martina Mauch, und Marlen Schumann, 1426. Münster/New York: Waxmann. https://doi.org/10.25656/01:18005.

Graf-Schlattmann, Marcel, Dorothee M. Meister, Gudrun Oevel, und Melanie Wilde. 2020. «Kollektive Veränderungsbereitschaft als zentraler Erfolgsfaktor von Digitalisierungsprozessen an Hochschulen». Zeitschrift für Hochschulentwicklung 15 (1): 19-39. https://doi.org/ 10.3217/zfhe-15-01/02.

Kalfa, Senia, Adrian Wilkinson, und Paul J Gollan. 2018. «The Academic Game: Compliance and Resistance in Universities». Work, Employment and Society 32 (2): 274-91. https://doi. org/10.1177/0950017017695043.

King, Emma, und Russell Boyatt. 2015. «Exploring Factors That Influence Adoption of E-Learning within Higher Education». British Journal of Educational Technology 46 (6): 1272-80. https://doi.org/10.1111/bjet.12195.

Kosmützky, Anna. 2018. «Managing Universities: Policy and Organizational Change from a Western European Comparative Perspective edited by Ivar Bleiklie, Jürgen Enders, and Benedetto Lepori». Comparative Education Review 62 (1): 151-54. https://doi.org/10.1086/695836.

Lai, Hui-Min, Yu-Lin Hsiao, und Pi-Jung Hsieh. 2018. «The Role of Motivation, Ability, and Opportunity in University Teachers' Continuance Use Intention for Flipped Teaching». Computers \& Education 124 (September): 37-50. https://doi.org/10.1016/j.compedu.2018.05.013.

Lin, Jingjing, und Lorenzo Cantoni. 2018. «Decision, Implementation, and Confirmation: Experiences of Instructors behind Tourism and Hospitality MOOCs». The International Review of Research in Open and Distributed Learning 19 (1). https://doi.org/10.19173/irrodl. v19i1.3402.

Long, Taotao, John Cummins, und Michael Waugh. 2019. «Investigating the Factors That Influence Higher Education Instructors' Decisions to Adopt a Flipped Classroom Instructional Model». British Journal of Educational Technology 50 (4): 2028-39. https://doi.org/10.1111/ bjet.12703.

Morgan, David L. 1996. «Focus Groups». Annual Review of Sociology 22 (1): 129-52. https://doi. org/10.1146/annurev.soc.22.1.129.

Musselin, Christine. 2007. «Are universities specific organisations». In Towards a multiversity? Universities between Global Trends and national Traditions, herausgegeben von G. Krücken, A. Kosmützky, und M. Torka, 63-84. Bielefeld: Transcript Verlag. https://doi. org/10.1515/9783839404683-004.

Reichertz, Jo. 2014. «Induction, Deduction, Abduction.» In The SAGE Handbook of Qualitative Data Analysis, herausgegeben von Uwe Flick, 123-35. Los Angeles: London: SAGE Publications Ltd.

Ryan, S. 2012. «Academic Zombies: A Failure of Resistance or a Means of Survival?». Australian Universities' Review, 54 (2): 3-11. https://aur.nteu.org.au/wp-content/uploads/2021/08/ AUR-54-02.pdf. 
Schneckenberg, Dirk. 2010. «Overcoming Barriers for ELearning in Universities - Portfolio Models for ECompetence Development of Faculty». British Journal of Educational Technology 41 (6): 979-991. https://doi.org/10.1111/j.1467-8535.2009.01046.x.

Stichweh, Rudolf. 2016. «Akademische Freiheit in europäischen Universitäten. Zur Strukturgeschichte der Universität und des Wissenschaftssystems». Die Hochschule: Journal für Wissenschaft und Bildung 25 (2): 19-36. https://www.hof.uni-halle.de/journal/texte/16_2/ Stichweh.pdf.

Schütz, Julia, Lena Rosenkranz, Bronwen Deacon, und Uwe Elsholz. i.E. «Never walk alone. Über das Verhältnis pädagogischer Professionalität und organisationaler Verantwortung an Hochschulen in Zeiten der Corona-Pandemie». In Things will never be the same again? Lehre und Hochschulentwicklung in Zeiten der Corona-Pandemie, herausgegeben von Holger Angenent, Jörg Petri und Tatiana Zimenkova. Bielefeld: Transcript Verlag.

Tippelt, Rudolf. 2009. «Erfahrungspotentiale durch vernetzte Organisationen. Zur Stärke dezentraler Beziehungen». In Organisation und Erfahrung: Beiträge der AG Organisationspädagogik, herausgegeben von Michael Göhlich, Susanne Maria Weber, und Stephan Wolff, 77-88. Wiesbaden: VS Verlag für Sozialwissenschaften. https://doi.org/10.1007/978-3-53191660-6_7.

Villa Braslavsky, Paula-Irene, Andrea Geier, und Ruth Mayer. 2021. "Das Sommersemester 2020 muss ein ,Nichtsemester' werden - Ein offener Brief aus Forschung und Lehre - \#nichtsemester». 2. August 2021. https://www.nichtsemester.de/cbxpetition/offener-brief/index. html.

Wannemacher, Klaus, unter Mitwirkung von Imke Jungermann, Sven Osterfeld, Julia Scholz, und Anna von Villiez. 2016. Organisation digitaler Lehre in den deutschen Hochschulen. Arbeitspapier 21, Hochschulforum Digitalisierung beim Stifterverband für die Deutsche Wissenschaft.

Weick, Karl E. 1976. «Educational Organizations as Loosely Coupled Systems». Administrative Science Quarterly 21 (1): 1-19. https://doi.org/10.2307/2391875.

Wilkesmann, Uwe. 2012. «Auf dem Weg vom Gelehrten zum abhängig Beschäftigten? Zwei deutschlandweite Surveys zur Lehrmotivation von Professoren». In Hochschule als Organisation, herausgegeben von Uwe Wilkesmann und Christian J. Schmid, 363-81. Organisationssoziologie. Wiesbaden: VS Verlag für Sozialwissenschaften. https://doi. org/10.1007/978-3-531-18770-9_21.

Wilkesmann, Uwe, und Christian Schmid, Hrsg. 2012. Hochschule als Organisation. Springer. https://doi.org/10.1007/978-3-531-18770-9.

Winde, Mathias, Said D. Werner, Barbara Gumbmann, und Solveigh Hieronimus. 2021. «Hochschulen, Corona und jetzt?». Wie Hochschulen vom Krisenmodus zu neuen Lehrstrategien für die digitale Welt gelangen. 28. September 2020. https://www.stifterverband.org/medien/hochschulen-corona-und-jetzt.

Wissenschaftsrat. 2021. "Impulse aus der COVID-19-Krise für die Weiterentwicklung des Wissenschaftssystems in Deutschland». https://www.wissenschaftsrat.de/download/2021/8834-21.pdf. 